\title{
EL TIEMPO Y LA POSIBILIDAD DE UN ENCUENTRO ENTRE HUSSERL Y DERRIDA
}

\author{
LUIS I. NIEL \\ Universidad Nacional del Litoral
}

\begin{abstract}
'Derrida está quizás más cerca de Husserl de lo que él pensaba" (David Wood)
\end{abstract}

El presente artículo tiene como intención partir de la problematización husserliana del tiempo y de la ulterior deconstrucción derrideana de la misma, para alcanzar y mostrar un punto de continuidad entre la obra de Husserl y la de Derrida. La idea central es plantear la posibilidad de una nueva fenomenología. En este sentido, son dos las metas a lograr. Por un lado, mostrar la vitalidad y posibilidad de la fenomenología de Husserl como una respuesta capaz de adaptarse a problemáticas propias de nuestra época, a partir de temas derrideanos tales como la temporalidad y lo otro, la escritura, etc. Por otro lado, mostrar primero, que la lectura de Derrida es una lectura fenomenológica, que puede entenderse en continuidad con la de Husserl y que incluso profundiza sus planteos, y segundo, que es una alternativa viable, no relativista y propicia para efectuar esta reconstrucción de la fenomenología. Como se anticipó, el hilo conductor de la presente lectura es el tiempo.

\section{El tiempo según la lectura fenomenológica de Husserl}

En el año 1901 aparecen publicadas las famosas Logische Untersuchungen de Husserl. Esta obra fue adquiriendo una profunda importancia en el mundo filosófico hasta convertirse en uno de los principales referentes de la tradición fenomenológica. Allí nos encontramos con la famosa refutación al psicologismo en los Prolegomena y con seis investigaciones que van desde una consideración propedéutica del lenguaje ( $1^{\mathrm{a}}$ investigación) a los problemas propios de una teoría fenomenológica del conocimiento ( $\sigma^{\mathrm{a}}$ investigación). En cuanto a la consideración con respecto al tiempo, Husserl reconoció que fue uno de los puntos que le faltó trabajar en esta obra. Pero en los años subsiguientes a su publicación la temporalidad se convertirá en una de sus más profundas preocupaciones llegando a ocupar un lugar central dentro de su filosofía. 
En realidad, consciente de la importancia de un trabajo detenido sobre el tiempo para una teoría del conocimiento, ya desde el año 1893 Husserl prestaba particular atención al estudio sobre la conciencia del tiempo inmanente (inneren Zeitbewußtsein). A esto lo atestiguan los 54 manuscritos editados por Rudolph Bernet ${ }^{1}$ que sumados a las famosas lecciones de 1905 organizadas por Edith Stein y editadas por Martin Heidegger $^{2}$, conforman en conjunto el tomo $\mathrm{X}$ de Husserliana ${ }^{3}$. Además habría que sumarle, y con particular atención, la reciente edición del tomo XXXIII de Husserliana que contiene los trabajos sobre la temporalidad de los años 1917-19184. Estos profundos trabajos marcarán el desarrollo ulterior de la fenomenología, fundamentalmente a partir de la así llamada nueva "fenomenología genética", manifestando el lugar axial que ocupa el tiempo en las meditaciones del Husserl maduro ${ }^{5}$.

Considerando ya su análisis del tiempo, vemos que el primer paso dado por Husserl es el de dejar fuera de juego toda consideración no trascendental sobre el mismo. Es decir, es puesto entre paréntesis el tiempo, en tanto tiempo del mundo o tiempo cósmico, para circunscribir el análisis

${ }^{1}$ Husserl Edmund, Texte zur Pbänomenologie des inneren Zeitbewußtsein 1893-1917, ed. Rudolph Bernet, Felix Meiner Verlag, Hamburg, 1985, (en adelante cito TPZB).

${ }^{2}$ Edmund Husserl, Vorlesungen zur Phänomenologie des inneren Zeitbewußtsein 1905, ed. Martin Heidegger, Max Niemayer Verlag, Tübingen, 2000, en adelante cito VPZB. Existe traducción española del mismo, Fenomenología de la conciencia del tiempo inmanente, trad. de Otto Langfelder, Ed. Nova).

${ }^{3}$ La primera parte del tomo X de Husserliana, editado por Rudolph Boehm, está conformada por las ya citadas lecciones editadas por Heidegger. La segunda parte son los textos reunidos bajo el nombre de "Ergänzende Texte zur Darstellung der Problementwicklung" (Textos complementarios para la exposición de la evolución del problema) que reúne los ya citados 54 manuscritos. Cf. el "Editorischer Berichl" de Rudolph Bernet, TPZB, p. LXIX-LXXIII.

+ Husserl Edmund, Die Bernauer Manuskripte über das Zeitbewnßstsein 1917-1918, ed. Rudolph Bernet y Dieter Lohmar, Husserliana XXXIII, Kluwer Academic Publishers, Dordrecht, 2001.

5 "Dentro de la fenomenología que une los nombres de Edmund Husserl y Martin Heidegger, la pregunta por el tiempo adquiere una posición conductora para la solución de la situación de las tareas fenomenológicas". (Friedrich-Wilhelm von Herrmann, Augustinus und die phänomenologische Frage nach der Zeit, Vittorio Klostermann, Frankfurt am Main, 1992, p. 15). 
fenomenológico al tiempo inmanente de la conciencia ${ }^{6}$. Esto es lo conocido con el nombre de Ausschaltung (neutralización, interrupción) der objektiven Zeit (del tiempo objetivo) ${ }^{7}$. Se neutraliza toda intuición trascendente para quedarse sólo con el dato hylético puro ${ }^{8}$; es decir, la intención fundamental es mantenerse en el terreno de las vivencias (Erlebnisse) subjetivas de la temporalidad'. No ya los objetos temporales trascendentes a la conciencia, es decir, las cosas del espacio, sino los objetos dados en la temporalidad inmanente de mi conciencia. Digo objetos, puesto que el mismo Husserl reconoce que no se puede hablar de la constitución del tiempo sin una referencia a la constitución de los objetos temporales ${ }^{10}$. La pregunta central que dirige no sólo las lecciones de 1905, sino en general también los manuscritos, es la de "cómo puede constituirse la objetividad temporal, es decir, la objetividad individual en general, en la conciencia subjetiva del tiempo"11. Es de esta idea de la circunscripción al tiempo de la conciencia, a partir de dónde Von Herrmann atribuye un claro paralelo entre Husserl y Agustín ${ }^{12}$.

El análisis de la temporalidad no se efectúa por lo tanto, en la exterioridad trascendente sino en lo que Husserl también denominaba el "flujo heraclíteo" de la conciencia ${ }^{13}$. Ya puesto entre paréntesis el mundo exterior, nos quedamos en la esfera interior de la conciencia. Ahora, la conciencia como tal no es algo estático y cerrado temporalmente sino que

\footnotetext{
${ }^{6}$ Una muy interesante discusión es la planteada por David Wood sobre si el tiempo objetivo es olvidado por Husserl o si en realidad hay una profunda intención de coordinar a ambos. Por razones de espacio no es posible abordar aquí tal problema. En tal sentido, cf. David Wood, The Deconstruction of Time, Northwestern University Press, Evanston, 2001, p. 59 y ss. De aquí en adelante cito "Wood".

$7 \Upsilon \mathrm{PZB}, \$ 1$ y $₫ 8$.

${ }^{8}$ Con esto no quiero ignorar la evolución de la noción de dato hylético en Husserl. Tal planteo excede los límites del presente trabajo.

9 "Los datos fenomenológicos son las aprehensiones temporales (Zeitauffassungen), las vivencias, en las que aparece lo temporal en sentido objetivo." (TPZB, \1). 10 “...así, un análisis fenomenológico del tiempo no puede explicar la constitución del tiempo sin referencia a la constitución de los objetos temporales." (VPZB, \7). ${ }^{11} \mathrm{VPZB}$, Introducción, p. 2.

${ }^{12}$ Mientras Aristóteles orienta su problematización en torno al tiempo objetivo de la naturaleza, Agustín lo hace en relación con el tiempo de la interioridad. Esto es trabajado en sus Confesiones, Libro XI. Cf. Von Herrmann, op. cit., p. 146-147.

13 "La conciencia es un constante flujo heraclíteo" (TPZB, No.51, p. 215).
} 
las vivencias se abren en este fluir llamado "heraclíteo"14. La fenomenología en cuanto tal, será la descripción de este flujo de vivencias, de su constante devenir temporal. Es importante aclarar que este fluir de la conciencia se constituye como tal a partir de la subjetividad trascendental que está más allá del tiempo y que es condición de posibilidad de tal fluir ${ }^{15}$. Debido a este motivo, cabe señalar una distinción importante a tener en cuenta, sobre la que no siempre se prestó debida atención, que es la realizada por Sokolowski entre las partes que proceden del objeto temporal (fase ahora, fase/s recién pasada/s y fase/s por venir) y las que proceden de la conciencia temporal (percepción ahora, memoria "primaria" y espera, o en la terminología posterior, impresión central o primaria, retención $y$ protención) ${ }^{16}$. Esta distinción se presenta como fundamental teniendo en cuenta la confusión que se tuvo durante mucho tiempo entre la conciencia trascendental constituyente del tiempo, o sea, que está más allá del tiempo y que es su condición de posibilidad, y la corriente del tiempo en la que se encuentran los objetos temporales ${ }^{17}$.

La subjetividad trascendental es por lo tanto, la condición de posibilidad del aparecer del objeto en el flujo temporal. Pero tal aparecer se da siempre en un punto (originario) de la percepción que es el ahora. "Es la

14 "Una inmanencia en la que nos encontramos constantemente perdidos. Este lugar de inmanencia, fue denominado por Husserl durante toda su vida flujo heracliteo de vivencias." (Jean-Michel Salanskis, Husserl, Les Belles Letres, Paris, 1998, p.17).

${ }^{15}$ Este fue un punto descuidado a la altura de las Logische Untersuchungen, pero que Husserl fue paulatinamente acentuando. Cf. Salanskis, p. 20.

${ }^{16}$ Sokolowski Robert, Husserlian Meditations. How Words Present Tbings, Northwestern University Press, Evanston,1989, p. 139.

${ }^{17}$ Sokolowski se cuenta a sí mismo como también a Seebohm y a Diemer, entre los que cayeron en tal error. Fue John Brough quien en "The Emergence of an Absolute Conciousness in Husserl's Early Writings on Time-Conciousness" desenmascaró tal error. Ibid., p.140, nota 4 . Una detenida lectura del $\$ 34$ de las Vorlesungen trae luz ante tal discusión con la distinción entre:

1) los objetos de la experiencia en el tiempo objetivo

2) el aparecer de la multiplicidad constituyente en sus diferentes niveles, la unidad inmanente del tiempo pre-empírico

3) la corriente de la conciencia absoluta constituyente del tiempo.

Los parágrafos subsiguientes (35-39) no hacen sino ratificar esta lectura de la notemporalidad de la conciencia constituyente del tiempo. (Cf. Bernet, Kern, Marbach, Edmund Husserl. Darstellung seines Denkens, Felix Meiner Verlag, Hamburgo, 1996, p. 103). 
subjetividad absoluta y tiene los atributos absolutos de algo que podría caracterizarse metafóricamente como «Flujo», que se origina en un punto de actualidad, punto fuente originario, «ahora», etc. En la vivencia de actualidad tenemos el punto fuente originario y una continuidad de momentos resonantes"18. Es sobre este punto de actualidad (Aktualitätspunkt), este originario punto fuente (Urquellpunkt), es decir, el abora (Jetzt), sobre el que tenemos que hacer hincapié para entender la ulterior crítica derrideana y sus consecuencias.

Como podemos vislumbrar, toda la lectura realizada por Husserl en torno al tiempo está profundamente marcada por su interés gnoseológico, en tanto que lo que le interesa es fundamentalmente el modo de darse del objeto inmanente a la conciencia en este flujo temporal. Por este motivo, su análisis del tiempo se encara a partir de los actos intuyentes inmediatos (unmittelbar anschauende Akte), denominados por Husserl, percepción (Wabrnebmung), recuerdo (Erinnerung) y espera (Erwartung). El lugar central a partir del cual se abre el horizonte temporal es la percepción presentante (gegenwärtigende Wabrnehmung), en la cual el objeto (interior) como tal es dado a la conciencia. Derrida no tardará en acentuar este aspecto de la fenomenología de Husserl acusándola de ser siempre una fenomenología de la percepción (presente) ${ }^{19}$.

Continuando con lo anteriormente afirmado, vemos que la percepción es el «punto fuente» a partir del cual Husserl abre el objeto al horizonte temporal ${ }^{20}$. Si bien es cierto que, a diferencia de Brentano, Husserl no niega realidad a la apertura temporal21, queda claro, y aquí es donde hace hincapié Derrida, que es el instante abora-presente el punto a partir del cual se centra

\footnotetext{
18 VPZB, p. 429.

19 Cf. Derrida Jacques, La voix et le phénomène. Introduction au problème du signe dans la phénoménologie de Husserl, PUF, París, 1967 (Trad. española de Patricio Peñalver, La voz y el fenómeno. Introducción al problema del signo en la fenomenología de Husserl, Pretextos, Valencia, 1995, p. 167. De aquí en adelante cito "LVF", con la paginación de la edición española.

20 "La percepción (Wabrnehmung) es la apercepción (Apperzeption), a través de la cual aparece el objeto como el mismo allí y ahora presente. La percepción adecuada presenta al objeto mismo." (TPZB, p. 39).

${ }^{21}$ Cf. TPZB, No. 14 y 15.
} 
la lectura de la temporalidad en Husserl. El dato dado por la percepción es lo que posibilita que haya retención ${ }^{22}$.

Todos estaríamos de acuerdo en afirmar la coherencia de Husserl en tal gesto, puesto que, en la teoría trascendental del conocimiento, de no haber un instante presente en el cual se de un dato para la percepción, no tendría sentido hablar de apertura a un horizonte de la temporalidad. Pero el problema es que aquí Derrida "percibe" un diáfano gesto de pertenencia a los imperativos propios de la metafísica de la presencia. "El pasado y el futuro siempre están determinados como presentes pasados o presentes futuros"23.

\section{La deconstrucción derrideana. El signo y la temporalidad}

Por lo general se suele desconocer el origen filosófico de uno de los más grandes filósofos franceses de los últimos años, Jacques Derrida. Sus primeros escritos atestiguan su claro origen dentro de la tradición fenomenológica. Como se verá este dato no es meramente anecdótico. A partir de trabajos menores como Le problème de la Génèse dans la philosophie de Husserl o de su más conocida introducción y traducción a L'Origine de la Géométrie de Husserl da sus primeros pasos en el mundo filosófico francés e internacional. Pero es en el año 1967 cuando irrumpe fuertemente con La voix et le phénomène, en donde inaugura las pautas fundamentales de ese modo de pensamiento luego denominado "deconstrucción», y en donde toma posición con relación a la fenomenología ${ }^{24}$. Éste constituye uno de los trabajos claves a la hora de una valoración positiva y futura de la fenomenología husserliana. La intención abierta en esta obra es desde un primer momento desenmascarar las más fundamentales e insospechadas limitaciones de la fenomenología a partir de la idea de «signo». Esta

22 "Si el contenido [dato de la percepción] nunca apareció en su presencia, no habría contenido para la retención al cual dirigirse en su ausencia." (John Brough, "Husserl and the Deconstruction of Time", en Review of Metaphysics 46, Marzo, 1993, p. 510).

${ }^{23}$ Derrida, Jacques, "Ousía y grammé", en Márgenes de la filosofía, trad. de Carmen González Marín, Cátedra, Madrid, 1998, p.68.

${ }^{2+}$ Habitualmente los epígonos de la deconstrucción han dejado de lado este texto revalorizando otros trabajos tales como De la gramatologie o L'écriture et la différence, también de 1967, olvidando que es precisamente aquí donde define las condiciones de su posición en relación con la filosofía trascendental husserliana, o quizás de la filosofia en general. 
"deconstrucción" del concepto de signo lleva a Derrida a plantear el problema en su relación con la temporalidad.

En la Introducción a LVF Derrida define cual será su recorrido y cual será su estrategia. El punto de partida es la noción de signo trabajada por Husserl en la $1^{\text {a }}$ de las Logische Untersuchungen (1901). De allí en adelante se encarga de mostrar cómo a partir de la conocida "actitud" reductiva que marca ya los primeros textos de Husserl ${ }^{25}$, el intento de eliminar lo extraño, o simplemente lo otro, lo lleva a caer en precisamente aquello de lo que pretende huir. Es decir, por un lado Derrida muestra que Husserl, en su búsqueda de pureza fenomenológica, intenta eliminar lo «no presente» a la conciencia trascendental, y que a través de este gesto ratifica la pertenencia del texto fenomenológico a la "metafísica de la presencia». Por otro lado, pretende mostrar que, a pesar de que tal ratificación de la presencia es la idea que dirige a todo el proyecto fenomenológico, si se es acorde con sus mismos pasos y "respetuoso con las cosas mismas", nos encontramos con una «no presencia originaria» que habita en su más íntimo y originario seno y que impide la absoluta identificación del presente consigo mismo (lo que Derrida denomina, "resistencia a la forma de presencia"). Resulta evidente a priori, el rol central que tiene la temporalidad dentro de la argumentación de Derrida.

El esquema es sucintamente el siguiente. Como nos muestra Derrida tanto en LVF como en la Introducción a L'Origine de la Géométrie, para Husserl la tematización del lenguaje es de propedéutica y fundamental importancia para el desarrollo de los problemas del conocimiento, puesto que es el substrato a partir del cual se erige todo posible significado ${ }^{26}$. El planteo fenomenológico necesita por lo tanto, una base ideal que garantice este discurso, y el signo, en tanto posibilidad ideal para la «repetición», cumple con tal cometido. La búsqueda de este substrato ideal comienza con

${ }^{25}$ Recordemos que Husserl hace explícitas referencias a la noción de "reducción" a partir de 1905.

${ }^{26}$ Cf. en especial Husserl Edmund, L'origine de la géométrie, traducido por Jacques Derrida, y en cuya introducción plantea claramente esta cuestión del lugar del lenguaje. "La explícita referencia al lenguaje como una instancia fundamental en la constitución histórica de las objetividades de la ciencia, lo presenta como condición de posibilidad de estas objetividades, y en este sentido como un "a priori" concreto." (Candioti María Elena, "Objetividad y constitución intersubjetiva", en Tópicos, No.7, Santa Fe, 1999, p.76-77). Cf. también, Wood, p. 41. 
las «distinciones esenciales» (wesentliche Unterscheidungen) ${ }^{27}$ que caracterizan al concepto de «signo» (Zeichen). Esta distinción es entre «expresión» (Ausdruck) y «señal» (Anzeichen). Siendo la señal un elemento extrínseco, indicativo, y por lo tanto, no siendo un constitutivo esencial del signo, la consideración fenomenológica procede a "ponerlo entre paréntesis» y así quedarse sólo con el elemento expresivo del signo. La expresión es tal, puesto que «significa» (bedeutet). Siguiendo las consideraciones etimológicas de Derrida, podemos decir que «quiere-decir» (veut-dire). Es por lo tanto, el «quererdecir» (Bedeutung/Vouloir-dire) el constitutivo ideal de la expresión y a donde Husserl quiere llegar.

Pero la exclusión fenomenológica no queda allí. Puesto que la (ex)presión conserva aún el elemento de la exterioridad, la pureza del análisis fenomenológico debe circunscribirse a la consideración de la Bedeutung en tanto querer-decir ideal de la consciencia a sí misma ${ }^{28}$. Aquí, en la "vida solitaria del alma"29, sostiene Derrida, se anticipa el motivo idealista husserliano de las Meditaciones cartesianas. Es sólo el significado ideal para una conciencia que no tiene que decir nada a nadie lo que tiene valor fenomenológico. Para suspender la relación con «un afuera», nos vemos remitidos a la interioridad de la conciencia, en donde "no hay que expresar nada". Para conservar su valor fenomenológico el signo debe permanecer dentro de la esfera interior de la conciencia.

$\mathrm{El}$ signo, puesto que dentro de la conciencia no tiene necesidad de comunicar nada (por lo expulsión de lo otro trascendente, y la limitación hacia aquello que es sólo para la conciencia trascendental), es sólo un signo representado. Es decir, siguiendo con la lectura de Derrida, cuando entra en acción la idea de representación ${ }^{30}$ el texto de Husserl entra a deconstruirse. Por un lado, la representación posibilita mantener una instancia ideal de repetición. Por otro lado es el punto en el que el discurso fenomenológico se fragmenta en su "pretensión metafísica» de conservar la presencia y

27 Husserl Edmund, 1901, Logische Untersuchungen, Felix Meiner Verlag, Hamburg, 1992, $2^{\circ}$ Tomo, $1^{a}$ Inv., Cap. 1, en Gesammelte Schriften 3. De aquí en adelante cito LU. Hay traducción española de la obra, Investigaciones lógicas, traducción de Manuel G. Morente y José Gaos, Alianza, Madrid, 1985.

${ }^{28}$ En este sentido es elocuente el nombre del tercer capítulo de LVF, titulado «El querer-decir como soliloquio».

29 “einsamen Seelenleben" (LU, $1^{2}$ Inv. \8.)

${ }^{30}$ Sea ya como Vorstellung, Vergegenwärtigung o Repräsentation. VF, p.98. 
desplazar la alteridad. «Aparece» ${ }^{31}$, lo que Derrida denomina, lo «no originario» que habita en el seno mismo de la originaria presencia.

Ahora, en consideración con el tema que nos concierne, vemos que el tiempo ocupa un lugar central dentro del trabajo husserliano sobre el signo puesto que es ostensible la referencia al valor temporal de la presencia ${ }^{32}$. El concepto de «ahora» (Jetat) se vuelve un punto de central referencia con relación a la consideración del signo y de la percepción. En este sentido, constatamos que, "el concepto de "ahora» del presente como puntualidad del instante autoriza discretamente, pero de manera decisiva, a todo el sistema de las "distinciones esenciales»"33. El ahora es el "punto fuente» en el que una percepción se da a una conciencia, constituyéndose de ese modo como principium y centro ineludible ${ }^{34}$. La temporalidad tiene como centro el presente del ahora, las demás fases temporales se articulan en función de éste ${ }^{35}$. Ahora, ¿qué lugar ocupa el signo en este esquema?

${ }^{31}$ No está de más recordar las precauciones heideggerianas de la utilización de tales términos que deberían ser tachados (o borrados, diría Derrida) puesto que lo otro como tal, no puede aparecer en el sentido de «hacerse presente».

32 "Este concepto del signo invoca un valor de presencia que sólo la concepción tradicional del tiempo puede sostener." (Wood, p. 270).

${ }^{33}$ LVF, p. 114.

34 "En el principio de todos los principios: que toda intuición (gebende Anschauung) originaria donante es una fuente de derecho del conocimiento Rechtsquelle der Erkenntnis); que todo lo que se nos es dado originariamente en la intuición (Intuition)... es un comienzo absoluto, un principium, llamado a ser fundamento, en el sentido genuino del término." (Husserl Edmund, 1913, Ideen zu einer reinen Pbänomenologie und phänomenologischen Philosopbie, Felix Meiner Verlag, Hamburg, 1992, en Gesammelte Schriften 5, $\ 24$, p. 51. Hay traducción española del mismo, Ideas relativas a una fenomenología pura y una filosofia fenomenológica, traducción de José Gaos, Fondo de Cultura Económica, México, 1992).

${ }^{35}$ No dejamos de tener en cuenta la postura de Natalie Alexander que sostiene que en Husserl hay numerosos elementos que subrayan el valor de la protención, y por lo tanto del futuro. El olvido derrideano de la protención sería, por lo tanto, no accidental, ya que hace hincapié en la percepción y su prolongación a través de la retención. "El mismo Derrida menciona a la protención sólo en dos oportunidades en La voz y el fenómeno... y la différance se desarrolla sólo en términos de retención." ALEXANDER, Natalie, "The Hollow Deconstruction of Time", en Derrida and Phenomenology, editado por William McKenna y J. Claude Evans, Contributions to Phenomenology, Vol. 20, p. 148. Lamentablemente la consideración de tal planteo excede el presente trabajo. 
El signo es aquello que en su carácter de representatividad hace presente (presentifica) aquello que está ausente (no-presente). Derrida señala una clara oposición entre el presente dado en una percepción, es decir, en una intuición originaria como tal ("principio de todos los principios" como instancia de no-significación ${ }^{36}$ ) en donde la ausencia y la inutilidad del signo se vuelven explícitas ${ }^{37}$, y el signo como no-presencia o presencia re-presentada. El esquema es el siguiente: percepción/presencia, signo/no-presencia/ presencia-representada (diferida) que se torna necesaria para la presentificación del pasado percibido ${ }^{38}$. El signo se vuelve un punto necesario de recurrencia como presentificación (Vergegenwärtigung, es decir, como representación) de lo ausente siendo de este modo posibilidad de reactivación de lo ya percibido para la constitución del discurso fenomenológico ${ }^{39}$. Desde el momento en que se admite en el seno mismo de la conciencia una apertura del presente hacia el horizonte de la temporalidad (por "respeto a las cosas mismas") se hace necesaria la referencia a un suplemento tal (signo) que se vuelve inexorablemente originario.

Cuando entra a jugar el valor de presencia-ausencia, y se vuelve inexorable la referencia a un contenido re-presentado en la conciencia, los supuestos "imperativos metafísicos" de Husserl comienzan a hacerse ostensiblemente explícitos y su pretensión entra a mancharse en su misma posibilidad. La presencia debe apelar a una ausencia (signo) que se abre en el horizonte del tiempo a partir de aquello que escapa al presente (différance). La percepción del ahora presente se diluye en tal apertura temporal y tiene necesidad de apelar a esta mentada no presencia para hacerse presente.

La relación con la representatividad está abierta cuando Husserl considera que el ahora no es algo cerrado en sí mismo sino abierto al horizonte de la temporalidad ${ }^{40}$. Esto ya puede observarse en los escritos del

${ }^{36}$ Cf. Infra, nota 34.

${ }^{37}$ Cf. LU, $\sigma^{\text {a }}$ Inv., $\int 26$.

38 Con esto no quiero negar otras posibilidades de la presentificación reconocidas por Husserl tales como la imaginación, etc.

39 “...del paso necesario de la retención a la re-presentación (Vergegenwärtigung) en la constitución de la presencia de un objeto (Gegenstand) temporal cuya identidad pueda ser repetida;" (LVF, p. 43).

40 "Que toda la realidad yace en el indivisible punto ahora (etrtpunkt), que todo en la fenomenología debería ser reducido a este punto, no son más que ficciones y conduce a absurdos." (TPZB, p. 35) 
joven Husserl cuando toma distancia con respecto a Brentano, alegando que éste privilegiaba el presente negando tangiblemente la posibilidad del pasado como algo real ${ }^{41}$.

En los derroteros del mismo planteo de Husserl, siguiendo fenomenológicamente la lectura de "las cosas mismas", Derrida se encuentra, por un lado, con una serie de presupuestos metafísicos que señalan la pertenencia de Husserl a los imperativos más propios de la metafísica de la presencia. El concepto de tiempo en cuanto tal es quien anuncia tal pertenencia. "El concepto de tiempo pertenece de parte a parte a la metafísica y nombra la dominación de la presencia"42. Pero, por otro lado, el concepto de tiempo es asimismo el punto a partir del cual la metafísica de la presencia entra a deconstruirse.

El punto-instante del ahora [momento (Augenblick) "indivisible" que establece el centro de referencia de esta fenomenología "de la percepción" de Husserl, tal como Derrida la define, «archi-forma» (Urform) de la conciencia], disuelve su imposible identidad en una necesaria remisión abierta y posibilitada por la horizontalidad del tiempo. La "seguridad metafísica" es sacudida por esta différance que nombra el tiempo ${ }^{43}$. El tiempo es a la vez el concepto que señala la pertenencia de todo discurso a la metafísica y el punto desde el cual toda metafísica se (auto) deconstruye.

La retención inaugura esta "duración" o "retardo" de la presencia del instante del ahora, ese desplazamiento inevitable que es a la vez condición de posibilidad y de imposibilidad del presente en su absoluta identificación ${ }^{44}$. Así, "en la retención, la presentación que da a ver, entrega un no-presente, un presente-pasado e inactual (...) Desde el momento en que se admite esta continuidad del ahora y del no-ahora, de la percepción y de la no-percepción en la zona de originariedad común a la impresión originaria y a la retención, se acoge lo otro en la identidad consigo del Augenblick: la no-presencia y la inevidencia en el parpadeo del instante"45.

${ }^{41}$ Cf. TPZB, Manuscritos 14 y 15, de ca. 1901. También la introducción de R. Bernet, p. XXIII.

+2 Derrida Jacques, "Ousía y gramme”, op.cit., p. 97.

+3 Sobre la noción de différance, cf. "La différance", en Márgenes de la filosofia, op.cit., o también puede verse la primera parte de De la gramatologia, SXXI, Buenos Aires, 1970.

H “...destruye radicalmente toda posibilidad de identidad consigo mismo en la simplicidad." (LVF, p. 119).

45 LVF, p. 118-119. 
Así alcanzamos junto con Derrida la lectura de la différance, que es sólo uno de los múltiples posibles nombres («archi-escritura», huella, fármacon, etc.) de aquel excedente de toda posible reapropiación conceptual filosófica (presente). La filosofía fenomenológica de Husserl se encuentra así ante un desbordamiento insuperable. $\mathrm{Y}$ a esto se llega, no saliendo de ella sino siguiendo conceptualmente tal lectura fenomenológica de las cosas mismas, en función de su relación con el tiempo. La crítica derrideana es de este modo no un planteo extrínseco a la lectura husserliana sino que acompaña fenomenológicamente la lectura de éste. En este sentido, Derrida sigue a Hegel cuando este afirma, "Si la refutación es fundamental, se toma y se desarrolla a partir de él [el principio, el comienzo] no a través de afirmaciones y ocurrencias efectuadas desde afuera" ${ }^{46}$.

\section{Pensar una nueva fenomenología. Entre Husserl y Derrida}

El problema planteado en la lectura derrideana de Husserl se erige en torno a la valoración del estatuto de la lectura deconstruccionista de tal texto. Habitualmente -aunque no en la totalidad de los casos- se ha valorado a la filosofía derrideana en su sentido estrictamente negativo. Es decir, entendiendo su lectura como una posición marcadamente relativista donde la diseminación textual se disuelve en una polisemia semántica y donde el «sentido» del texto «deconstruido» desaparece en una infinitud de sentidos posibles. A partir de tal planteo se infiere directamente que Derrida aniquila no sólo a la fenomenología de Husserl y sino en general a toda la filosofía, tildándola de mera "metafísica de la presencia" y quedando como resultado sólo un juego de textos en donde ninguna nueva producción filosófica parecería ya posible. ¿Es tal el planteo de Derrida?

Mi respuesta es rotunda y necesariamente negativa. Según la interpretación del presente artículo, Derrida no anunciaría como sostiene Éric Alliez ${ }^{47}$ la muerte de la fenomenología, o dicho más genéricamente la muerte de toda filosofía en su pretensión de trascendentalidad, sino que más bien conduciría a la fenomenología, siguiendo sus mismos pasos para mostrarle sus propias limitaciones. En este sentido, me apropio de las palabras de Natalie Depraz cuando sostiene que la intención de Derrida "no es tornar

t6 Hegel G.W.F., Phänomenologie des Geistes, Hauptwerke, Tomo 2, Felix Meiner Verlag, Hamburgo, 1999, p.21.

47 Alliez Éric, De l'impossibilité de la phénoménologie. Sur la philosophie française contemporaine, Librairie Philosophique J. Vrrin, Paris, 1995. 
caduco y nulo el método fenomenológico y deshacerse de él definitivamente, sino por el contrario, su intención es traerlo justamente allí a donde pueda aún tener un sentido, producir una inteligibilidad filosófica" 48 .

Creo que interpretar de un modo positivo a la obra de Derrida, no sólo es más fructífero en el sentido programático y desiderativo, sino que se sigue de la lectura de sus mismos textos ${ }^{49}$. Hay en Derrida un profundo respeto y amor por la letra y el texto husserliano del tiempo. La indagación no se realiza en un plano extrínseco sino que parte de los mismos presupuestos y de los mismos conceptos de Husserb". Pero superarla no en el sentido dejarla atrás sino para mostrar aquel punto en que es desbordada, que es a partir del concepto del tiempo. Derrida busca claramente en las condiciones de posibilidad de la fenomenología el punto en que se abre su condición de imposibilidad como planteo absoluto $y$ clausurante. Pero que estos pasos dados son claramente fenomenológicos, se ve en la continuidad existente entre una y otra lectura (de Husserl y de Derrida), y que intentó plasmarse a partir del análisis del tiempo, en los dos primeros capítulos de este artículo.

Por eso, cuando David Wood se pregunta si el planteo de Derrida tiene pretensiones de trascendentalidad ${ }^{51}$, mi respuesta sería categóricamente que sí. Si bien es cierto que tal pretensión está planteada por el mismo Derrida en términos que condicen con su deconstrucción, tales como los de «cuasi-trascendentalidad» ${ }^{52}$, no obstante, en su lectura del tiempo, la búsqueda de las condiciones de posibilidad de tal aparecer a partir de la retención y su consecuente desbordamiento del ahora presente, dejan en claro que el «cuasi» no destruye su pretensión de fondo, y por qué Derrida no es un relativista, ni pretende disolver la filosofía en la literatura ${ }^{53}$. "Algo que he

\footnotetext{
${ }^{+8}$ Depraz N., "Introduction", en Derrida et la phénoménologie, Alter, Paris, 2000, p. 9.

t9 Esto al menos en el marco acotado en que se propone la presente lectura, que es el de la lectura de los textos de Husserl, pero que, estoy convencido, una profundización de otras obras, incluso las posteriores, aseverarían tal posición. La cuestión excede el propósito del presente artículo.

50 "Es a partir de lo fenomenológico mismo de donde él intenta superar a la fenomenología." (Depraz, op.cit., p. 9).

${ }^{51}$ Wood, p. 270.

52 Derrida Jaques, Deconstrucción y pragmatismo, trad. de Marcos Mayer, Paidós, Buenos Aires, 1998, p.158.

${ }^{3}$ Ibidem, "...jamás traté de confundir literatura y filosofía o de reducir la filosofía a la literatura", p.155.
} 
aprendido de las grandes figuras de la historia de la filosofía, de Husserl en particular, es la necesidad de formular preguntas trascendentales para no quedar atrapados en la fragilidad de un incompetente discurso empirista y, por lo tanto, para evitar el empirismo, el positivismo y el psicologismo, es que resulta interminablemente necesario renovar el cuestionamiento trascendental'54.

Sin embargo, Derrida es consciente, a diferencia de Heidegger o al menos de cierta lectura de él ${ }^{55}$, que no hay una posible salida de la metafísica. Que mientras nos movamos dentro de la conceptualidad propia de la metafísica de la presencia, es decir, de la filosofía, inexorablemente nos vemos atrapados por ella. En tal sentido coincido con Wood cuando afirma que según Derrida no hay salida de la filosofía ${ }^{56}$. Ahora, su planteo renovador, como él mismo lo reconoce, consiste en moverse dentro del marco de la ruptura, de lo olvidado, o de lo dejado de lado, es decir, en hacer filosofía desde los márgenes, surcando con el mismo trazo las condiciones de posibilidad de un discurso y sus condiciones de imposibilidad. "Pero este cuestionamiento [trascendental] debe renovarse tomando en cuenta la posibilidad de la ficción, de lo accidental y de la contingencia, asegurando así que esta nueva forma de cuestionamiento trascendental sólo imita al fantasma de la clásica seriedad trascendental sin renunciar a aquello que, dentro de ese fantasma, constituye un legado esencial”. Y agrega más abajo, que es necesario "definir la condición trascendental de posibilidad como una condición de imposibilidad" 57 .

Tal persecución de Derrida, creo, es una clara búsqueda fenomenológica de neta inspiración husserliana, que podríamos denominar «fenomenología de los márgenes». Una nueva fenomenología que da un paso más, y que por lo tanto no desatiende a aquel excedente que habita lo más íntimo de la realidad. En este sentido es fundamental la problematización del tiempo, ya que es el punto en que la conceptualización

${ }^{5+}$ Ibid., p. 159. La cursiva es mía.

55 "A diferencia de Heidegger (al menos de cierto textos de temprana data), Derrida niega la posibilidad de la recuperación que repite este pasado y por eso mismo niega también la posibilidad de una superación de la metafísica." (Bernet Rudolph, "Die ungegenwärtige Gegenwart. Anwesenheit und Abwesenheit in Husserls Analyse des Zeitbewußtseins", en Zeit und Zeitlichkeit bei Husserl und Heidegger, Verlag Karl Alber, Freiburg/München, 1983, Phänomenologische Forschungen, Tomo 14, p.21).

56 "Derrida es el primero en admitir que no hay un escape real de la filosofía." (Wood, p. 269).

${ }^{57}$ Deconstrucción y pragmatismo, op.cit., p. 158. 
metafísica dirigida por el valor de presencia se desplaza impidiendo su absoluta identidad. El tiempo es la infinita apertura a lo otro. Lo otro que no es un "más allá" al que podría arribarse tras superaciones dialécticas, sino que habita la misma presencia, siendo su condición de posibilidad. Esto permite replantear a la fenomenología, sin salir de ella, “... la fenomenología debe reconocer, en las experiencias cruciales de la temporalidad y del otro, que la no presencia a sí de la representación en el recuerdo y de la apresentación en la relación con el alter ego parece ser tan originaria y constituyente como la presencia a sí viviente... él [Husserl] no ve en la muerte, la condición misma de la vida" 58 .

Se trata así, de “... una lucha de un tipo muy distinto, en la que el vencedor y el vencido están uno en el interior del otro, una lucha que tiene lugar sobre la frontera que, al mismo tiempo separa y une la filosofía de la no filosofía, en la cesura, in dem Riß, que al mismo tiempo rompe la presencia y la torna posible"59. En esta nueva «fenomenología de los márgenes», o «fenomenología de lo otro», el tiempo no es una mera ficción o un artificio literario escrito como epitafio de la muerte de la filosofía o de la metafísica. En esta nueva lectura interior a la misma filosofía, que sigue respetuosamente el imperativo fenomenológico del "amor por las cosas mismas", vemos que el tiempo es el punto en el cual se nos muestra que "la cosa misma siempre se sustrae" y que la realidad nos desborda de un modo tal que nunca se agota en su totalidad por lo captable en términos intencionales inteligibles para una conciencia trascendental. Tal es la causa por la cual aún seguimos filosofando. Pensar desde la clausura de la filosofía no es pensar la muerte de la filosofía, todo lo contrario, es pensar en su siempre renovable riqueza, es pensar en sus concretas posibilidades futuras.

"Comenzar a pensar su clausura, es decir, también su futuro" 60.

58 Dastur Françoise, "Finitude et répétition chez Husserl et Derrida", en Derrida et la phénoménologie, ed.cit., p.48.

${ }^{59}$ Dastur, p. 51.

60 Derrida Jacques, "La phénoménologie et la clôture de la metaphysique. Introduction à la pensée de Husserl”, en Derrida et la phénoménologie, ed.cit., p. 84. 


\title{
Resumen
}

El artículo propone una línea de interpretación que articula el pensamiento de Husserl con el de Derrida a partir de la lectura que ambos realizan sobre el tiempo. Siguiendo esta línea, la intención es alcanzar un punto de continuidad entre la obra de estos pensadores, y plantear tal continuidad en términos fenomenológicos. En una primera instancia se pretende mostrar la vitalidad y posibilidad de la fenomenología de Husserl como una respuesta capaz de adaptarse a problemáticas propias de nuestra época, en particular desde su trabajo sobre la temporalidad y en conexión con la interpretación de la misma realizada por Derrida. En un segundo momento, mostrar que la lectura de Derrida es una lectura fenomenológica que se desarrolla en continuidad con la de Husserl y que incluso profundiza sus planteos. En tal sentido, entenderla como una alternativa viable, no relativista y propicia para efectuar esta nueva interpretación de la fenomenología. En síntesis, siguiendo el hilo conductor del análisis del tiempo, la idea central a la que se apunta es plantear la posibilidad de una fenomenología que articule los planteos de Husserl y de Derrida y que podría denominarse «fenomenología de los márgenes».

\begin{abstract}
This article proposes a line of interpretation which connects Husserl's with Derrida's thought by means of the reading of time that both philosophers make. Following this line, the intention is to reach a point of continuity between the work of these two thinkers, and therefore to sustain such continuity into phenomenological terms. In the first place, it pretends to show the vitality and possibility of Husserl's phenomenology as an answer capable to adapt to the problems of our own time, in particular from his work about temporality and in connection with Derrida's interpretation of this concept. And secondly, is our intention to show that the Derridean reading can be considered as a phenomenological reading developed itself in continuity with Husserl's and which even deepens on the phenomenological proposals. In this way, it can be understood as viable, non-relativistic and as a proper alternative to carry out a new interpretation of phenomenology. In summary, following the common thread of time analysis, this article is focused in setting the possibility of articulating Husserl's and Derrida's phenomenology, which could be called "phenomenology of marges".
\end{abstract}

